\title{
Non-invasive Monitoring of Core Body Temperature for Targeted Temperature Management in Post- Cardiac Arrest Care
}

Kyle Fiorini ( $\sim$ kfiorini@uwo.ca )

London Health Sciences Centre https://orcid.org/0000-0002-6788-5136

Tanya Tamasi

LHSC: London Health Sciences Centre

Justin Dorie

LHSC: London Health Sciences Centre

Ahmed F. Hegazy

LHSC: London Health Sciences Centre

Ting-Yim Lee

LHSC: London Health Sciences Centre

Marat Slessarev

LHSC: London Health Sciences Centre

\section{Research Articles}

Keywords: Monitoring of Core Body, Post-Cardiac Arrest Care, esophageal probe

Posted Date: September 30th, 2021

DOl: https://doi.org/10.21203/rs.3.rs-927179/v1

License: (9) (i) This work is licensed under a Creative Commons Attribution 4.0 International License.

Read Full License

Version of Record: A version of this preprint was published at Frontiers in Medicine on April 13th, 2022.

See the published version at https://doi.org/10.3389/fmed.2022.810825. 


\section{Abstract}

\section{Background}

Accurate monitoring of core body temperature is integral to targeted temperature management (TTM) following cardiac arrest. However, there are no reliable non-invasive methods for monitoring temperature during TTM. We compared the accuracy and precision of a novel non-invasive Zero-Heat Flux Thermometer (SpotOnä) to a standard invasive esophageal probe in a cohort of patients undergoing TTM post cardiac arrest.

\section{Methods}

We prospectively enrolled 20 patients undergoing post-cardiac arrest care in the intensive care units at the London Health Sciences Centre in London, Canada. A SpotOnä probe was applied on each patient's forehead, while an esophageal temperature probe was inserted, and both temperature readings were recorded at 1-minute intervals for the duration of TTM. We compared the SpotOnä and esophageal monitors using Bland-Altman analysis and Pearson correlation, with accuracy set as a primary outcome. Secondary outcomes included precision and correlation. Bias exceeding $0.1^{\circ} \mathrm{C}$ and limits of agreement exceeding $0.5^{\circ} \mathrm{C}$ were considered clinically important.

Results

Sixteen ( $80 \%)$ of patients had complete data used in the final analysis. The median (interquartile range) duration of recording was 38 (12-56) hours. Compared to the esophageal probe, SpotOnä had a bias of $0.05 \pm 0.35^{\circ} \mathrm{C}$ and $95 \%$ limits of agreement of -0.64 to $0.74^{\circ} \mathrm{C}$. Pearson correlation coefficient was 0.98 (95\% confidence interval 0.9796-0.9805), with a two-tailed p-value of $<0.0001$.

\section{Conclusion}

The SpotOnä is an accurate method that may enable non-invasive monitoring of core body temperature during TTM, although its precision is slightly worse than the pre-defined $0.5^{\circ} \mathrm{C}$ when compared to invasive esophageal probe.

\section{Introduction}

Targeted Temperature Management (TTM) is an important component of intensive care unit (ICU) management in post-cardiac arrest patients. TTM protocols are associated with improved neurologic outcomes in cardiac arrest survivors (1,2), and generally consist of rapid cooling, maintenance, rewarming and hyperthermia prevention stages delivered over 24-72 hours following cardiac arrest (35). Recent evidence also suggests that targeted normothermia and prevention of hyperthermia in the post-cardiac arrest period may be as effective as hypothermia in improving patient outcomes (6). Accurate measurement of core body temperature is critical to ensuring successful implementation of 
these protocols, and current guidelines recommend the use of invasive esophageal, nasopharyngeal, bladder, endotracheal cuff or pulmonary artery temperature sensors (3-5).

A recently developed non-invasive surface temperature monitor (SpotOn ${ }^{\mathrm{TM}}, 3 \mathrm{M}$, Minnesota, USA) may provide a non-invasive alternative for tracking core temperature during TTM. It uses zero-heat-flux technology (7) based on creating an isothermic tunnel under the measurement site by insulating heat loss from the skin surface and allowing it to equilibrate with core body temperature using an internal heating element. This monitor showed good correlation, accuracy and precision compared to esophageal, nasopharyngeal and pulmonary artery sensors in perioperative settings (8-10), and performed better than bladder temperature sensors during cardiac surgery (10). However, these studies only measured temperatures for up to 9 hours and never compared the performance of the SpotOn ${ }^{\mathrm{TM}}$ monitor to invasive core body temperature monitors during longer durations and wider temperature ranges used in TTM protocol (which can last up to 72 hours post-cardiac arrest).

The aim of this study was to assess the accuracy, precision, and correlation of the SpotO ${ }^{\text {TM }}$ monitor compared to standard invasive core body temperature monitor in cardiac arrest patients receiving TTM.

\section{Methods}

\section{Study Design and Patients}

This was a prospective observational single center study that recruited consecutive cardiac arrest patients admitted to two of the intensive care units at London Health Sciences Centre in London, Canada. Inclusion criteria were: adult patients 18 years and older with in- and out-of-hospital cardiac arrest who qualified for TTM as per established hospital protocols developed in accordance with current practice guidelines (3). Exclusion criteria were: contraindications to TTM, immediate plan to withdraw lifesustaining measures, inability to obtain consent, skin rash or infection over the forehead, or medical tape allergy. This study was approved by Western University Health Sciences Research Ethics Board (protocol \# 109432). We obtained signed informed consent from all patient substitute decision makers prior to commencing study procedures.

\section{Study Procedures}

Following study enrollment, we simultaneously measured patient's core temperatures using SpotOn ${ }^{\mathrm{TM}}$ and esophageal temperature probes for the duration of the TTM protocol that included cooling, maintenance, rewarming and hyperthermia prevention (up to 72 hours following cardiac arrest). In each patient, we used pressure-sensitive adhesive to secure the $\mathrm{SpotO}^{\mathrm{TM}}$ temperature probe to the patient's forehead as per the product monograph. We then connected the probe to the SpotOn ${ }^{T M}$ central portable console and initiated temperature measurement. All patients had esophageal temperature probes inserted as per our institutional TTM care protocol. We used an automated data capture module (MediCollector $\rightarrow$, USA) to simultaneously record temperatures from both the SpotOn ${ }^{T M}$ and esophageal temperature probes at 1minute intervals for the duration of the TTM protocol (up to 72 hours following study enrollment). We 
used case report forms for each patient to record demographic data, type of cardiac arrest, comorbidities, duration of TTM protocol and temperature recording from the medical chart.

\section{Data analysis and statistics}

Statistical analysis was performed using GraphPad Prism version 8.3 (GraphPad Software $\rightarrow$, San Diego, CA, U.S.A.). Continuous variables were reported using medians and interquartile ranges (IQRs) for nonnormally distributed data or means and standard deviations for normally distributed data, and categorial variables were reported as frequencies (\%). The primary endpoint for this study was accuracy between the SpotOn ${ }^{T M}$ and esophageal temperature probes, measured as bias using Bland-Altman analysis. Secondary outcomes include precision, measured as limits of agreement using the Bland-Altman analysis, and correlation, measured using the Pearson correlation coefficient.

\section{Results}

We enrolled 20 consecutive patients in the study. Four (20\%) patients had incomplete recording due to failure of recording equipment and were excluded from data analysis. The remaining 16 patients (5 female) were included in the final analysis (Fig. 1). Their clinical characteristics are summarized in Table 1. Median (IQR) age was 66 years $(54,72)$ years, $9(56 \%)$ patients had an out-of-hospital cardiac arrest, $4(25 \%)$ had a shockable rhythm, 8 (50\%) arrested in pulseless electrical activity, and the three most common comorbidities were hypertension (56\%), dyslipidemia (38\%) and diabetes (31\%). The median (IQR) duration of the TTM protocol was 24 (24-24) hours and the median recording time was 38 (12-56) hours. Figure 2 shows a sample recording from a representative patient. 
Table 1

Patient Demographics

\begin{tabular}{|ll|}
\hline Demographics & Number \\
\hline Subjects & 16 \\
\hline Sex (M:F) & $11: 5$ \\
\hline Age (IQR) & $65.5(54-71.5)$ \\
\hline IHCA & $7(43.8 \%)$ \\
\hline OHCA & $9(56.2 \%)$ \\
\hline VT or VF & $4(25 \%)$ \\
\hline PEA or Asystole & $12(75 \%)$ \\
\hline Cooling - Hours (IQR) & $24(24-24)$ \\
\hline Recording - Hours (IQR) & $38.14(11.69-56.10)$ \\
\hline Comorbidities & \\
\hline Coronary Disease & $0(0 \%)$ \\
\hline CHF & $3(18.75 \%)$ \\
\hline Diabetes & $5(31.25 \%)$ \\
\hline Hypertension & $9(56.25 \%)$ \\
\hline CKD & $3(18.75 \%)$ \\
\hline Dyslipidemia & $6(37.5 \%)$ \\
\hline COPD & $2(12.5 \%)$ \\
\hline
\end{tabular}

Combining data across patients, we had 28,685 sets of data points from the two probes. For our primary outcome (accuracy), the bias measured using Bland-Altman analysis was $0.05 \pm 0.35^{\circ} \mathrm{C}$ (Fig. 3). For our secondary outcomes, the precision as measured by Bland-Altman analysis $95 \%$ limits of agreement was -0.64 to $0.74{ }^{\circ} \mathrm{C}$ (Fig. 3). The correlation analysis between the two modalities showed a Pearson coefficient of 0.9801 ( $95 \%$ confidence interval 0.9796 to 0.9805 ), with a two-tailed $p$-value of $<0.0001$ (Fig. 4).

\section{Discussion}

Targeted temperature management is a fundamental component of post-cardiac arrest care and relies upon accurate and reliable core temperature monitoring (3-5). In order to reliably measure core temperature, TTM guidelines recommend esophageal, nasopharyngeal, bladder, endotracheal cuff and pulmonary artery temperature sensors $(3-5)$. However, these monitors are invasive and may not be 
readily available in all centres. Given that a bias of less than $0.1^{\circ} \mathrm{C}(11)$ and a $95 \%$ limits of agreement of less than $0.5^{\circ} \mathrm{C}$ (9) between two temperature monitors were considered acceptable in prior studies, our results suggest that the SpotOn ${ }^{\text {TM }}$ monitor has acceptable accuracy and correlation, though is just outside the acceptable margin of precision when compared to the esophageal probe for monitoring core temperature during TTM.

Although the SpotOn ${ }^{\mathrm{TM}}$ thermometer has been shown to be a reliable tool for continuous temperature monitoring in perioperative settings (8-10), our study is the first to assess its performance across a wider range of temperatures and longer durations of monitoring encountered during TTM. In contrast to current invasive core temperature monitors, SpotOn ${ }^{\text {TM }}$ is non-invasive, easy to apply, and provides an indirect measure of brain temperature. Given that the purpose of TTM is to limit secondary brain injury by accurately titrating brain temperature, indirect monitoring of brain temperature may be more clinically relevant than monitoring of core body temperature at non-brain sites. In the future, selective brain cooling while maintaining body normothermia may enable neurologic benefits of TTM while minimizing systemic side effects associated with whole-body cooling (12). In these cases, indirect monitoring of brain temperature using SpotOn ${ }^{\mathrm{TM}}$ would be highly relevant. The only potential drawbacks of the SpotOn ${ }^{\mathrm{TM}}$ device include restrictions with application in patients with adhesive allergies and potential for skin breakdown with extensive use, although these adverse events were not seen in our study, nor in a prior study that used SpotOn ${ }^{\text {TM }}$ for the duration of time similar to TTM protocols (13).

Our study had several limitations. We enrolled only 20 patients at a single-centre and 4 patients were excluded due to a failure of data logging equipment. While the remaining 16 patients is a small sample size, long duration and high frequency of temperature recording resulted in a large dataset spanning a broad range of temperatures, enabling a robust comparison between two temperature monitoring modalities. While the "gold standard" for temperature monitoring is considered the pulmonary artery catheter (14), we compared SpotO $\mathrm{n}^{\text {TM }}$ performance against the esophageal temperature probe since pulmonary artery catheters are no longer used routinely in post-cardiac arrest patients in our units. The precision and accuracy of the esophageal temperature probe relative to the pulmonary artery catheter have been previously demonstrated in another study (14). We therefore chose to use esophageal measurement as a safer reference method in our study.

\section{Conclusion}

The SpotOn ${ }^{\mathrm{TM}}$ monitor is an accurate method for continuous temperature monitoring in post-cardiac arrest patients, although its precision is slightly worse than the pre-defined $0.5^{\circ} \mathrm{C}$ when compared to the invasive esophageal probe. This device can be used as an alternative to invasive monitors to measure core temperature during targeted temperature management. Furthermore, the possibility of indirect brain temperature measurement warrants further investigation.

\section{Declarations}


Ethics approval and consent to participate was approved by Western University Health Sciences Research Ethics Board (protocol \# 109432). We obtained signed informed consent from all patient substitute decision makers prior to commencing study procedures. All authors have consented to publication.

The authors have no competing or conflicting interests. We have not received any additional funding. Each author contributed to the paper. Specifically, authors TT, JD, and MS were responsible for data collection. KF, MS, and AH performed the data analysis. KF and MS wrote the first draft of the manuscript, and all authors contributed to the review and revisions of the manuscript, as well as the final draft approval.

\section{References}

1. Hypothermia after Cardiac Arrest Study Group (2002) Mild therapeutic hypothermia to improve the neurologic outcome after cardiac arrest. N Engl J Med 346(8):549-56.

2. Bernard SA, Gray TW, Buist MD, Jones BM, Silvester W, Gutteridge G, Smith K (2002) Treatment of comatose survivors of out-of-hospital cardiac arrest with induced hypothermia. N Engl J Med 346(8):557-63.

3. Howes D, Gray SH, Brooks SC, Boyd JG, Djogovic D, Golan E, Green RS, Jacka MJ, Sinuff T, Chaplin T et al (2016) Canadian Guidelines for the use of targeted temperature management (therapeutic hypothermia) after cardiac arrest: A joint statement from The Canadian Critical Care Society (CCCS), Canadian Neurocritical Care Society (CNCCS), and the Canadian Critical Care Trials Group (CCCTG). Resuscitation 98:48-63.

4. Callaway CW, Donnino MW, Fink EL, Geocadin RG, Golan E, Kern KB, Leay M, Meurer WJ, Peberdy MA, Thrompson TM et al (2015) Part 8: Post-Cardiac Arrest Care: 2015 American Heart Association Guidelines Update for Cardiopulmonary Resuscitation and Emergency Cardiovascular Care. Circulation 132(18):S465-82.

5. Nolan JP, Soar J, Cariou A, Cronberg T, Moulaert VRM, Deakin CD, Bottiger BW, Friberg H, Sunde K, Sandroni C (2015) European Resuscitation Council and European Society of Intensive Care Medicine Guidelines for Post-Resu scitation Care 2015. Resuscitation 95:202-222.

6. Dankiewicz J, Cronberg T, Lilja G, Jakobsen JC, Levin H, Uljén S, Rylander C, Wise MP, Oddo M, Cariou A et al (2021) Hypothermia versus Normothermia after Out-of-Hospital Cardiac Arrest. NEJM 384:22832294.

7. Fox RH, Solman AJ, Isaacs R, Fry AJ, MacDonald IC (1973) A new method for monitoring deep body temperature from the skin surface. Clin Sci 44(1):81-6.

8. Eshraghi Y, Nasr V, Parra-Sanchez I, Van Duren A, Botham M, Santoscoy T, Sessler DI (2014) An Evaluation of a Zero-Heat-Flux Cutaneous Thermometer in Cardiac Surgical Patients: Anesth Analg 
119(3):543-9.

9. Iden T, Horn E-P, Bein B, Böhm R, Beese J, Höcker J (2015) Intraoperative temperature monitoring with zero heat flux technology (3M SpotOn sensor) in comparison with sublingual and nasopharyngeal temperature: An observational study. Eur J Anaesthesiol 32(6):387-91.

10. Mäkinen M-T, Pesonen A, Jousela I, Päivärinta J, Poikajärvi S, Albäck A, Salimen U, Pesonen E (2016) Novel Zero-Heat-Flux Deep Body Temperature Measurement in Lower Extremity Vascular and Cardiac Surgery. J Cardiothorac Vasc Anesth 30(4):973-8.

11. Sessler D (2008) Temperature Monitoring and Perioperative Thermoregulation. Anesthesiology 109:318-338.

12. Fazel Bakhsheshi M, Keenliside L, Lee TY (2018) A novel selective cooling system for the brain: feasibility study in rabbits vs piglets. ICMx 6(45).

13. Bräuer A, Fazliu A, Perl T, Heise D, Meissner K, Florian Brandes I (2020) Accuracy of zero-heat-flux thermometry and bladder temperature measurement in critically ill patients. Sci Rep 10:21746.

14. Lefrant JY, Muller L, Emmanuel de La Coussaye J, Benbabaali M, Lebris C, Zeitoun N, Mari C, Saïssi, Ripart J, Eledjam J (2003) Temperature measurement in intensive care patients: comparison of urinary bladder, oesophageal, rectal, axillary, and inguinal methods versus pulmonary artery core method. Intensive Care Med 29:414-418.

\section{Figures}




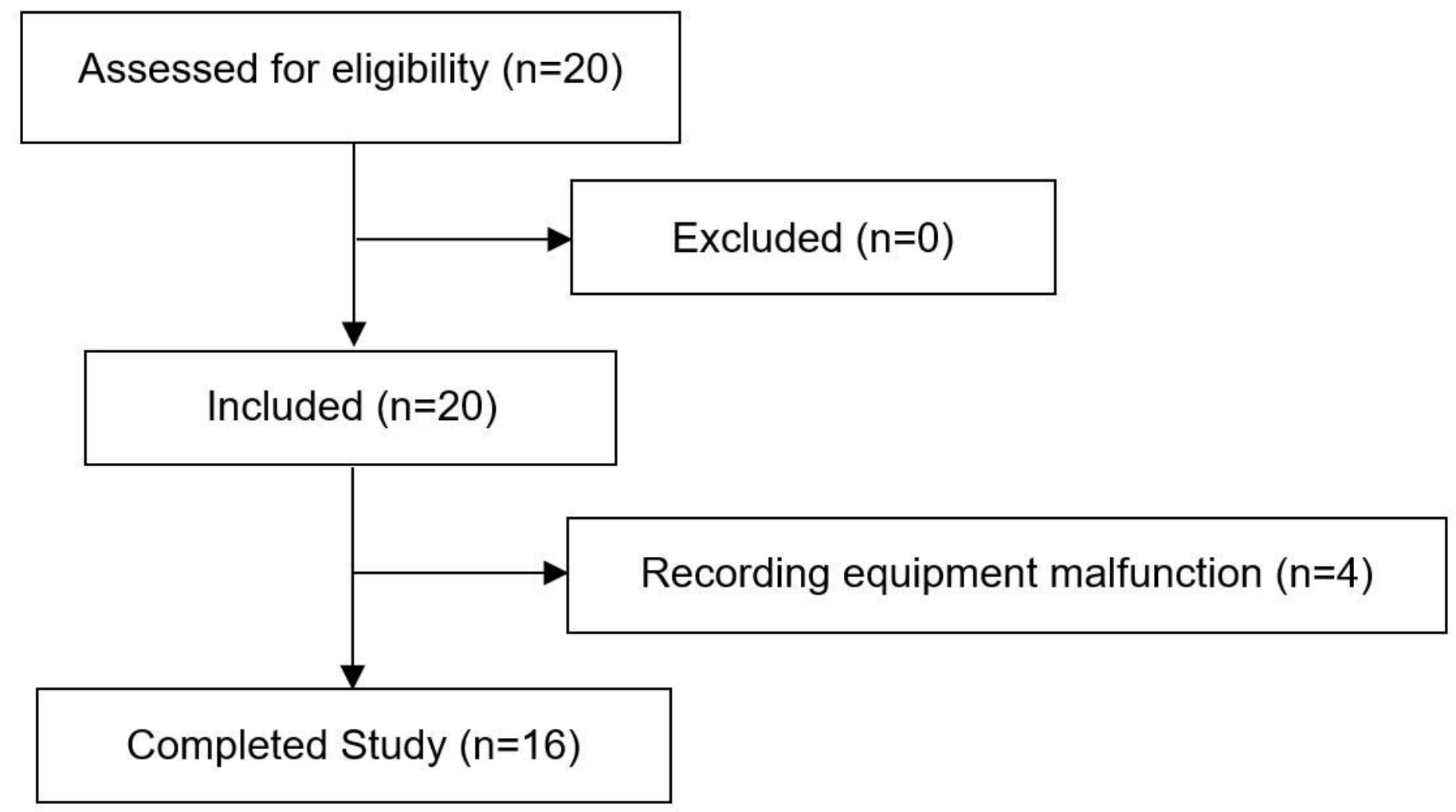

Figure 1

Enrolment Flow Diagram

\section{Temperature vs Time: Subject \#5}

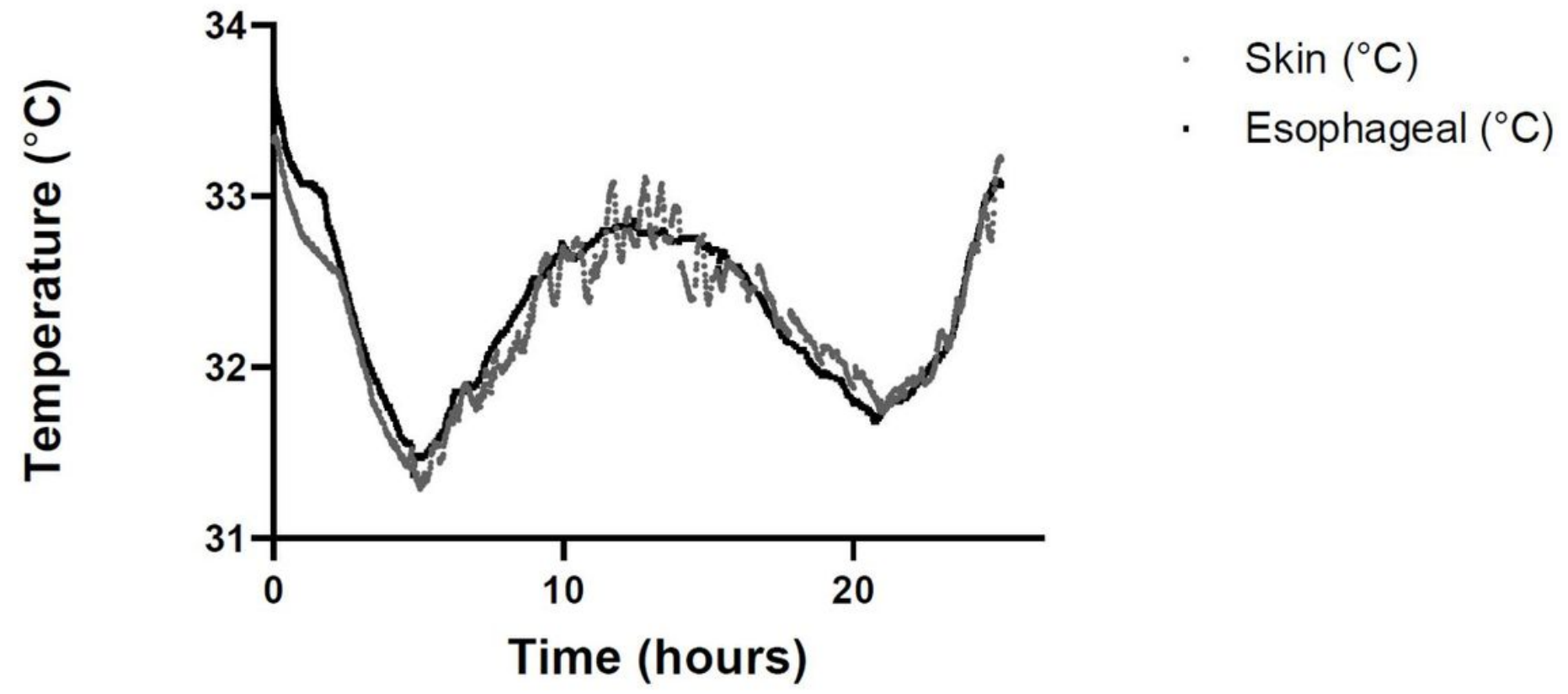

Figure 2 
Representative Patient Temperature Measurement between Skin and Esophageal Probes

\section{Bland-Altman}

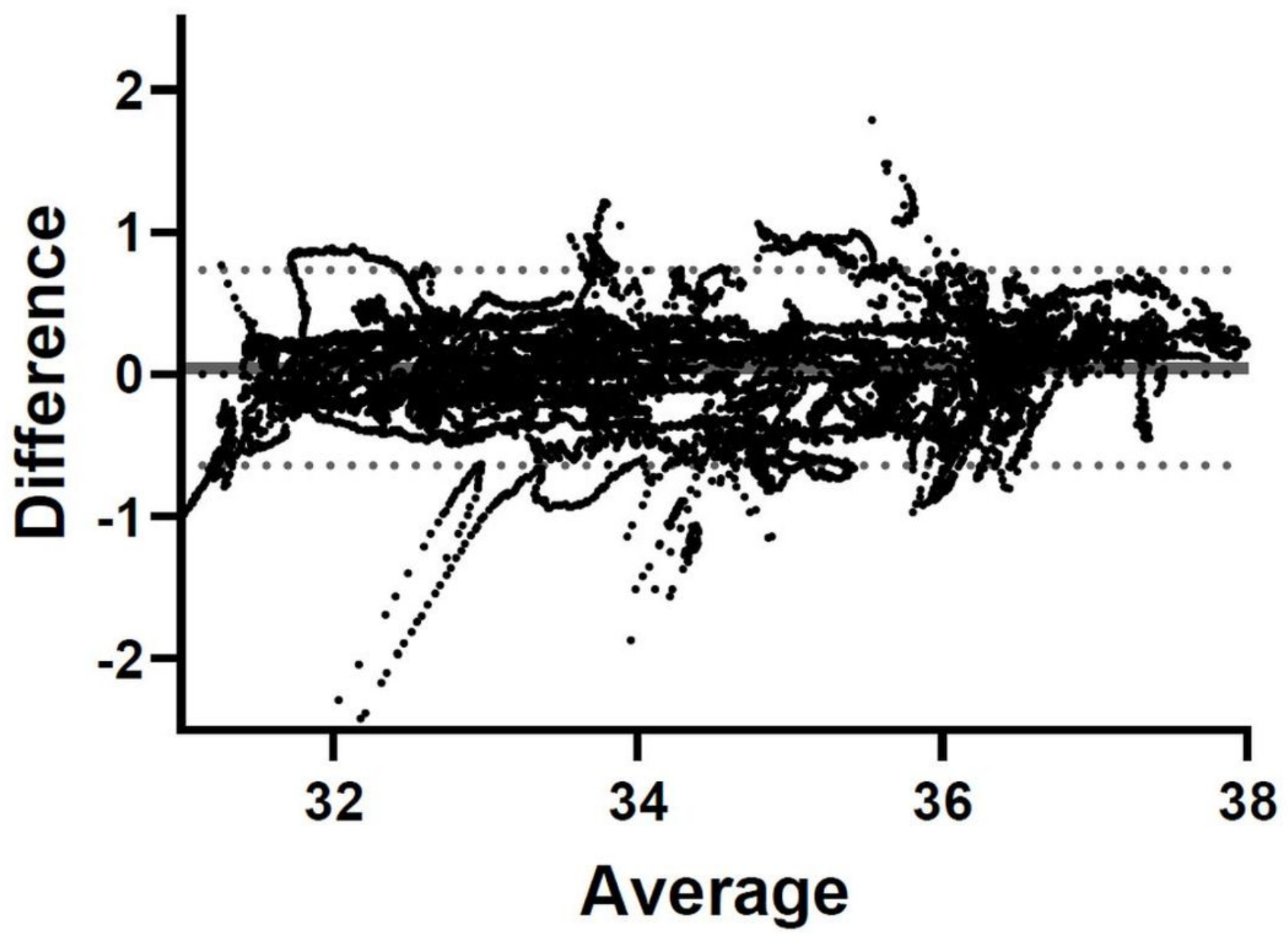

Figure 3

Bland-Altman Analysis comparing Skin and Esophageal Probes 


\section{Correlation}

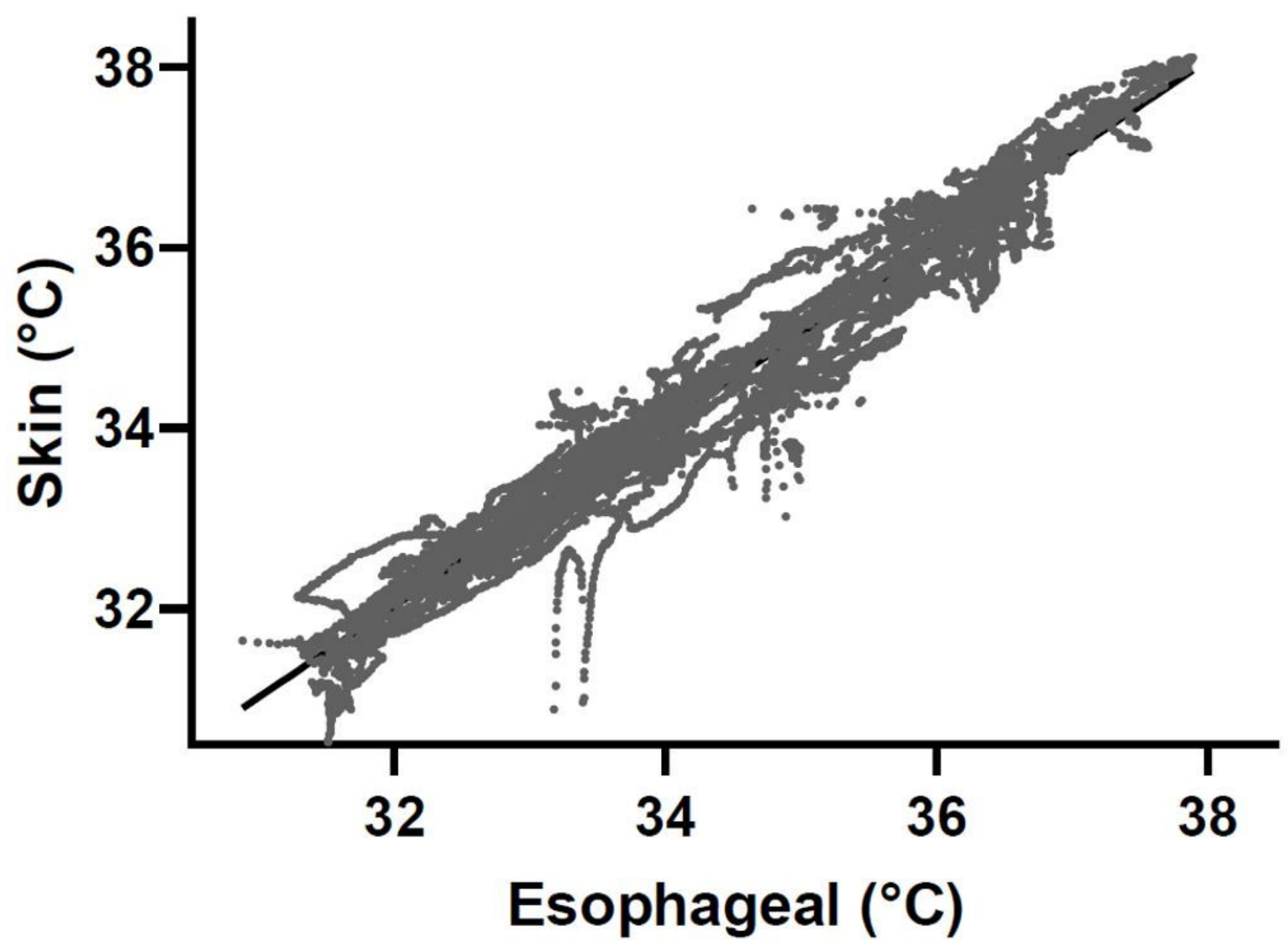

Figure 4

Correlation between Skin and Esophageal Probes 